\title{
FERTILIZANTE DE LIBERAÇÃO LENTA NO DESENVOLVIMENTO DE MUDAS DE Eucalyptus grandis
}

\author{
Überson Boaretto Rossa ${ }^{1}$, Alessandro Camargo Angelo ${ }^{2}$, Itamar Antonio Bognola ${ }^{3}$, \\ Danielle Janaina Westphalen ${ }^{4}$, Jaçanan Eloisa de Freitas Milani ${ }^{4}$ \\ ${ }^{1}$ Instituto Federal Catarinense, Araquari, Santa Catarina, Brasil - boarettorossa@ gmail.com \\ ${ }^{2}$ Universidade Federal do Paraná, Departamento de Ciências Florestais, Curitiba, Paraná, Brasil - alessandrocangelo@gmail.com \\ ${ }^{3}$ Empresa Brasileira de Pesquisa Agropecuária, Colombo, Paraná, Brasil- iabog @ yahoo.com.br \\ ${ }^{4}$ Universidade Federal do Paraná, Programa de Pós-Graduação em Engenharia Florestal, Curitiba, Paraná, Brasil - \\ daniellejanaina76@gmail.com; jacanan.milani@gmail.com \\ Recebido para publicação: 21/03/2013 - Aceito para publicação: 23/05/2014
}

\begin{abstract}
Resumo
Uma das ações mais importantes para aumentar a produção de mudas de essências florestais é a fertilização do substrato. A utilização de fertilizante de liberação lenta (FLL) pode contribuir para a obtenção de mudas de melhor qualidade. O objetivo do trabalho foi avaliar doses crescentes de FLL e fertilizante convencional (FC), bem como comparar esses fertilizantes no desenvolvimento de mudas de Eucalyptus grandis. O estudo foi realizado na região do Vale do Itajaí, SC. Os tratamentos foram a adição de FLL e FC para cada experimento nas seguintes doses de formulado: T1 - $0 \mathrm{~kg}$ (testemunha); T2 - 2 kg; T3 - 4 kg; T4 - 6 kg; T5 - 8 kg e T6 - 10 kg.m ${ }^{-3}$ de substrato-base. Decorridos 174 dias da semeadura, foram analisadas as variáveis altura total, diâmetro do colo, biomassa fresca da parte aérea, biomassa seca da parte aérea, biomassa seca da raiz, biomassa seca total, dose de máxima eficiência técnica e teores de nutrientes da parte aérea das mudas de cada tratamento. Em todos os tratamentos houve resposta positiva no desenvolvimento das mudas, entretanto as mudas tiveram melhor crescimento sob doses entre 9,1 e $12,9 \mathrm{~kg} . \mathrm{m}^{-3}$ de fertilizante de liberação lenta.

Palavras-chave: Qualidade de mudas; nutrição de mudas; vivericultura; fertilização; substrato.
\end{abstract}

\begin{abstract}
Slow release fertilizer in development of seedlings of Eucalyptus grandis. One of the most important actions to increase production of seedlings is substrate fertilization. The use of slow release fertilizer (FLL) can contribute to the achievement of best seedlings. The aim of this research was to evaluate increasing doses of FLL and FC, as well as compare these fertilizers in developing seedlings of Eucalyptus grandis. The study was conducted in Vale do Itajai, Santa Catarina State. The treatments were the addition of FLL and HR in each experiment in the following doses: T1 - $0 \mathrm{~kg}$ (control), T2 $2 \mathrm{~kg}$; T3 $-4 \mathrm{~kg}, \mathrm{~T} 4-6 \mathrm{~kg}$; T5 - $8 \mathrm{~kg}$ and T6 - $10 \mathrm{~kg} \cdot \mathrm{m}^{-3}$ of base substrate. After 174 days of sowing, the variables analyzed were total height, stem diameter, fresh weight of shoot, shoot dry biomass, root dry weight, total biomass and maximum dose of technical efficiency, and nutrient levels of the seedlings of each treatment. In all treatments there was positive response in seedling development, however, the plants had better growth in doses ranging from 9.1 to $12.9 \mathrm{~kg} . \mathrm{m}^{-3}$ of slow release fertilizer.
\end{abstract}

Keywords: Seedling quality; seedlings nutrition; cultivation of seedlings; fertilization; substrate.

\section{INTRODUÇÃO}

O sucesso de um povoamento florestal tem demandado o desenvolvimento de pesquisas que otimizem a produção de mudas selecionadas com características ideais de desenvolvimento, evitando-se assim, segundo José et al. (2005), mudas de baixo padrão de qualidade e com menor potencialidade de incrementos, com perspectivas de menores ganhos de volume em madeira. Além disso, procura-se evitar que as mudas se desenvolvam com tendência de menor uniformidade e pior qualidade do fuste, a fim de não onerar os custos de implantação (CARNEIRO, 1995). No Brasil, a madeira de espécies do gênero Eucalyptus vem se constituindo em uma das principais fontes de matéria-prima para as indústrias de base

FLORESTA, Curitiba, PR, v. 45, n. 1, p. 85 - 96, jan. / mar. 2015.

Rossa, U. B. et al.

ISSN eletrônico 1982-4688 / ISSN impresso 0015-3826

DOI: $10.5380 /$ rf.v45i1.31224 
florestal, devido à alta capacidade de adaptação em diferentes ambientes e seu rápido crescimento (TRUGILHO et al., 2007).

Fonseca (2000) indicou que a obtenção de mudas de qualidade pode ser alcançada de maneira prática, rápida e fácil pela observação dos parâmetros morfológicos. Os principais parâmetros morfológicos que determinam a qualidade das mudas são a altura, o diâmetro do colo e o peso da parte aérea e das raízes (CARNEIRO, 1995). Tais parâmetros podem também ser utilizados para calcular relações que definem o índice de qualidade de muda, como proposto por Dickson et al. (1960). O índice é obtido pela proporcionalidade da biomassa seca total com a soma das proporcionalidades entre a altura e o diâmetro do caule e entre a biomassa seca dos tecidos aéreos e subterrâneos, resultando na ponderação desses parâmetros para verificação da qualidade de muda (LANG, 1998; FONSECA et al., 2002). Os parâmetros de ordem fisiológica, por exigirem medições complexas, podem ficar em segundo plano (MARQUES, 2004).

Em viveiros é comum o uso de substratos pobres em nutrientes ou desequilibrados nutricionalmente, ocasionando baixa qualidade das mudas, comprometendo seu desenvolvimento em campo (CECONI et al., 2007).

A adoção de técnicas de fertilização do substrato é uma das práticas mais importantes para obtenção de expressivos aumentos no crescimento e qualidade de mudas de essências florestais (BRONDANI et al., 2008).

Conforme Gonçalves e Poggiani (1996), a necessidade de adubação decorre do fato de que nem sempre o substrato é capaz de fornecer todos os nutrientes que as plantas precisam para um adequado crescimento. Entre as técnicas de fertilização do substrato em viveiros florestais, o emprego de fertilizantes de liberação lenta representa umas das mais viáveis e racionais alternativas (BOCKMAN; OLFS, 1998; SHAVIV, 1999). Como principal desvantagem, os fertilizantes de liberação lenta apresentam custo superior ao das fontes solúveis, requerendo a adequação das doses nos diferentes sistemas de produção, visando otimizar o uso do insumo e garantir a produção econômica de mudas (SCIVITTARO et al., 2004). Entretanto a utilização de adubos de liberação lenta reduz problemas de excesso de solubilidade, perdas por lixiviação de nutrientes (BARBIZAN et al., 2002) e a mortalidade de plantas por choque de plantio (LANG, 2008).

Esses fertilizantes incluem compostos solúveis no seu interior (NPK e alguns micronutrientes), envolvidos por uma membrana semipermeável que, por efeito da temperatura, dilata e contrai, controlando a liberação gradual e osmótica de nutrientes ao substrato (BENNETT, 1996), mantendo constantes os níveis dos elementos essenciais para as mudas durante todo o período de crescimento (JOSÉ et al., 2009). Dessa maneira, apresenta evidentes vantagens sobre os fertilizantes convencionais em diversas culturas, como arroz, hortícolas e ornamentais (HEFNER; TRACY, 1991; CSIZINSZKY, 1994), em diferentes tipos de solo, climas e manejos. Entretanto, para espécies florestais de interesse ambiental, os estudos da ação dos fertilizantes de liberação lenta, bem como as doses de maior eficiência técnica, ainda são incipientes.

Diante do exposto, o presente trabalho teve como objetivo avaliar o crescimento inicial de mudas de Eucalyptus grandis submetidas a doses crescentes de fertilizante de liberação lenta (FLL) e fertilizante convencional (FC), bem como comparar esses fertilizantes no desenvolvimento dessas mudas.

\section{MATERIAL E MÉTODOS}

O presente trabalho foi realizado durante o período de setembro de 2008 a março de 2009, sendo conduzido em viveiro de produção de mudas localizado sob as seguintes coordenadas geográficas: $27^{\circ} 11^{\prime} 16^{\prime \prime}$ S e 49³9'37' W, em uma altitude média de $701 \mathrm{~m}$ em relação ao nível do mar, instalado no Instituto Federal de Educação, Ciência e Tecnologia, no município de Rio do Sul, SC.

As sementes de Eucalyptus grandis foram adquiridas da empresa Klabin Paraná Papéis, oriundas de pomar de primeira geração, instalado no município de Telêmaco Borba, PR.

As sementes foram semeadas em tubetes canônicos com 6 estrias de $53 \mathrm{~cm}^{3}$, utilizando-se como substrato-base uma mistura de substrato florestal Plantmax (60\%), composto orgânico peneirado (30\%) e vermiculita de granulometria média (10\%). Suas características químicas e físicas estão apresentadas na tabela 1. Para os tratamentos, utilizou-se fertilizante de liberação lenta de marca comercial Basacote ${ }^{\circledR}$ Mini 6M, com formulação13-6-16 $\left(\mathrm{N}^{-} \mathrm{P}_{2} \mathrm{O}_{5}-\mathrm{K}_{2} \mathrm{O}\right)+$ micronutrientes e fertilizante convencional na formulação 5-20-10 $\left(\mathrm{N}-\mathrm{P}_{2} \mathrm{O}_{5}-\mathrm{K}_{2} \mathrm{O}\right)$. 
Tabela 1. Análise química e física dos componentes do substrato utilizados para a produção de mudas de Eucalyptus grandis com dosagens crescentes de fertilizantes de liberação lenta e convencional.

Table 1. Chemical and physical analysis of the substrate components for production of Eucalyptus grandis seedlings under increasing of slow-release fertilizer and conventional doses.

\begin{tabular}{|c|c|c|c|c|c|c|c|}
\hline \multicolumn{2}{|l|}{ Composto orgânico } & \multicolumn{2}{|c|}{ Plantmax $^{\circledR}$} & \multicolumn{2}{|c|}{ Fertilizante de liberação lenta } & \multicolumn{2}{|c|}{ Fertilizante convencional } \\
\hline $\mathrm{pH}\left(\mathrm{H}_{2} \mathrm{O}\right)$ & 6,3 & $0 \mathrm{H}(\mathrm{H} \Omega)$ & $58(1+05)$ & $\mathrm{N}(\%)$ & 13,00 & $\mathrm{~N}(\%)$ & 5,00 \\
\hline Índice (SMP) & 6,8 & $\mathrm{pH}($ & 5 & $\mathrm{P}_{2} \mathrm{O}_{5}[$ Sol. em & & $\mathrm{P}_{2} \mathrm{O}_{5}[$ Sol. em & \\
\hline $\mathrm{Ca}\left(\mathrm{cmol}_{\mathrm{c}} \cdot \mathrm{dm}^{3}\right)$ & 7,0 & Capacidade de & & $\left.\mathrm{CNA}+\mathrm{H}_{2} \mathrm{O}\right](\%)$ & 6,00 & $\left.\mathrm{CNA}+\mathrm{H}_{2} \mathrm{O}\right](\%)$ & 20,00 \\
\hline $\operatorname{Mg}\left(\mathrm{cmol}_{\mathrm{c} .} \mathrm{dm}^{3}\right)$ & 4,4 & retenção de & 150 & $\mathrm{~K}_{2} \mathrm{O}(\%)$ & 16,00 & & \\
\hline $\mathrm{Al}\left(\mathrm{cmol}_{\mathrm{c}} \cdot \mathrm{dm}^{3}\right)$ & 0,0 & água $(\%)$ & & $\mathrm{MgO}(\%)$ & 1,40 & $\mathrm{~K}_{2} \mathrm{O}[\mathrm{So}$ & 1000 \\
\hline $\mathrm{H}+\mathrm{Al}\left(\mathrm{cmol}_{\mathrm{c}} \cdot \mathrm{dm}^{3}\right)$ & 1,7 & & & $\mathrm{~S}(\%)$ & 10,00 & $\left.\mathrm{H}_{2} \mathrm{O}\right](\%)$ & 10,00 \\
\hline $\mathrm{CTC}\left(\mathrm{cmol}_{\mathrm{c} .} \mathrm{dm}^{3}\right)$ & 16,5 & Umidade (\%) & $0^{c}$ & $\mathrm{~B}(\%)$ & 0,02 & & \\
\hline Saturação Al (\%) & 0,0 & & & $\mathrm{Cu}(\%)$ & 0,05 & & \\
\hline Saturação base (\%) & 89,5 & Densidade & 450 & $\mathrm{Fe}(\%)$ & 0,26 & $\mathrm{Ca}(\%)$ & 9,00 \\
\hline M.O. $(\%)$ & 7,8 & & & $\operatorname{Mn}(\%)$ & 0,06 & & \\
\hline Argila (\%) & 27 & Condutividade & & $\operatorname{Mo}(\%)$ & 0,015 & & \\
\hline$P\left(\mathrm{mg} \mathrm{dm}^{3}\right)$ & 560 & elétrica & $2,6(+/-0,3)$ & diâmetro grânulos (mm) & 1,5 a 2,8 & $\mathrm{~S}(\%)$ & 8,00 \\
\hline $\left.\mathrm{K}(\mathrm{mg} \mathrm{dm})^{3}\right)$ & 1160 & $(\mathrm{mS} . \mathrm{cm})$ & & peso de 1.000 grãos $(\mathrm{g})$ & 9,58 & & \\
\hline
\end{tabular}

Para mistura dessas matérias-primas, bem como para a homogeneização das doses testadas ao substrato base, foi utilizada betoneira por um período de 5 minutos. Os tubetes foram preenchidos e logo submetidos a mesa compactadora por 10 segundos, objetivando densidade uniforme do substrato. Após a semeadura, as bandejas com os vasos foram mantidas com nível de sombreamento de $50 \%$ e a umidade do substrato foi mantida por sistema de irrigação por microaspersão.

Para possibilitar análise comparativa entre os tratamentos (FLL e FC), foram instalados dois experimentos, adotando-se delineamento inteiramente casualizado, com seis tratamentos em quatro repetições, tendo 40 plantas como unidade experimental. Os tratamentos foram: $\mathrm{T} 1-0 \mathrm{~kg}$ (testemunha); T2 - $2 \mathrm{~kg} ; \mathrm{T} 3-4 \mathrm{~kg} ; \mathrm{T} 4-6 \mathrm{~kg}$; T5 - $8 \mathrm{~kg}$ e T6 - $10 \mathrm{~kg}$ de FLL e FC por m³ de substrato-base.

Aos 30 dias da semeadura, foi efetuado o raleio, eliminando-se as mudas excedentes em cada tubete, deixando apenas a que apresentava maior vigor e que estava mais centralizada. Houve ainda reposição de mudas não sobreviventes após o raleio.

Decorridos 174 dias da semeadura, coletaram-se dados de altura da parte aérea da muda, medindo-se do nível do solo até o ápice. $\mathrm{O}$ diâmetro do colo mediu-se a $0,5 \mathrm{~cm}$ do substrato. Em seguida, determinou-se a biomassa fresca da parte aérea com balança de precisão milesimal. As raízes foram separadas do solo manualmente e lavadas em peneiras de $2 \mathrm{~mm}$, para evitar possíveis perdas de raízes. As amostras da parte aérea e de raízes foram acondicionadas em sacos de papel pardo e secas em estufa a $60{ }^{\circ} \mathrm{C}$, com ventilação forçada até atingir peso constante.

Foram analisados os parâmetros biométricos de altura total (H), diâmetro do colo (DC), biomassa fresca da parte aérea (BFPA), biomassa seca da parte aérea (BSPA), biomassa seca da raiz (BSR) e biomassa seca total (BST). Os índices de qualidade de muda analisados foram a relação entre altura e diâmetro do colo (H/DC) e o índice de qualidade de Dickson (IQD) (DICKSON et al. 1960). Após verificação dessas variáveis, foi calculada a dose de máxima eficiência técnica (DMET), a partir da equação de regressão para cada variável estudada em função da dose de FLL e FC aplicada. Ao nível de $1 \%$ de probabilidade e pelo coeficiente de determinação $\left(\mathrm{R}^{2}\right)$, foi ajustada equação de $2^{\circ}$ grau para relacionar as doses de adubação com as variáveis de interesse.

A análise química de tecido foi realizada no Laboratório de Biogeoquímica e Nutrição de Plantas (LABINP) do Setor de Ciências Agrárias da UFPR. Para o preparo das amostras, utilizaram-se as mesmas plantas secas, moídas em moinho tipo Willey, deixando a amostra com partículas menores ou iguais a $0,5 \mathrm{~mm}$.

A análise química total de $\mathrm{P}, \mathrm{K}, \mathrm{Ca}, \mathrm{Mg}, \mathrm{Fe}, \mathrm{Mn}, \mathrm{Cu}$ e $\mathrm{Zn}$ foi efetuada por digestão via seca, após incineração em mufla a $500{ }^{\circ} \mathrm{C}$ e posterior solubilização em $\mathrm{HCl}_{3}$ mol. $\mathrm{L}^{-1}$. O P foi determinado por colorimetria com vanadato-molibdato de amônio (cor amarela) e leitura em espectrofotômetro UV/VIS. A determinação de $\mathrm{K}$ e $\mathrm{Na}$ foi realizada por fotometria de emissão, e de $\mathrm{Ca}, \mathrm{Mg}, \mathrm{Fe}, \mathrm{Mn}, \mathrm{Cu}$ e $\mathrm{Zn}$, por 
espectroscopia de absorção atômica por chama. Todas as análises foram realizadas de acordo com a metodologia proposta por Martins e Reissmann (2007).

As determinações de carbono e nitrogênio total e a relação $\mathrm{C} / \mathrm{N}$ foram feitas em equipamento analisador CNHS, da marca Elementar, modelo Vario El, sendo que para as determinações analíticas as amostras secas e moídas foram submetidas ao moinho mecânico e passadas em peneiras de 1,00 mm. Cerca de aproximadamente $20 \mathrm{mg}$ de amostra foram pesadas em balança analítica específica e acondicionados em cápsulas de estanho, para serem levadas ao analisador.

\section{RESULTADOS E DISCUSSÃO}

\section{Qualidade das mudas}

Os resultados obtidos indicam um aumento em mais de quatro vezes no crescimento da altura em função da adubação, confirmando a grande importância da adubação na melhoria da qualidade da muda e seu desenvolvimento vegetal (MARSCHNER, 1995). A resposta à adubação apresentou ajuste quadrático (Figura 1), tendo como dose de máxima eficiência técnica (DMET) o valor de 11,80 kg.m $\mathrm{m}^{-3}$.

A altura é uma característica importante quando há a necessidade de plantio das mudas em áreas colonizadas por plantas daninhas, onde a altura da espécie de interesse leva a maior competitividade por luz. Por isso, em muitos viveiros florestais, a altura é considerada para o estabelecimento do valor das mudas na ocasião da comercialização ao silvicultor, sendo usualmente utilizada para estimar seu padrão de qualidade (GOMES et al., 2002). Ainda, é um dos mais importantes parâmetros para estimar o crescimento no campo (REIS et al., 1991; PARVIAINEN, 1981), considerando que sua medição não é destrutiva, é de fácil execução e tecnicamente aceita como uma boa medida do potencial de desempenho das mudas (MEXAL; LANDS, 1990).

Moraes Neto et al. (2003a) verificaram resultado semelhante em mudas de $E$. grandis produzidas em tubetes, com altura média de $45,2 \mathrm{~cm}$ aos 125 dias de semeadura, fertilizadas com FLL na formulação $14 \% \mathrm{~N}-14 \% \mathrm{P}_{2} \mathrm{O}_{5}-14 \% \mathrm{~K}_{2} \mathrm{O}$, com dosagem de $6,42 \mathrm{~kg} \cdot \mathrm{m}^{-3}$. Ou seja, dosagem inferior ao valor de $11,8 \mathrm{~kg} \cdot \mathrm{m}^{-3}$, observada em nosso trabalho.

Aos 90 dias do plantio, Freitas et al. (2005) perceberam maior crescimento em mudas de E. grandis $(32,7 \mathrm{~cm})$ e E. saligna $(32,6 \mathrm{~cm})$ adubados com FLL, produzidas com diferentes tipos de substratos em bloco prensado.

Uma mesma tendência foi observada em trabalho realizado por Brondani et al. (2008), sendo encontrado o ponto máximo em altura na dose de FLL de $2,7 \mathrm{~kg} \cdot \mathrm{m}^{-3}$ para a espécie Anadenanthera colubrina (angico-branco).

Todas as mudas que receberam doses de FLL apresentaram altura superior às que receberam FCC, apontando para a importância de uma contínua disponibilização dos nutrientes durante o período de desenvolvimento da muda.

Embora exista estreita semelhança entre os trabalhos científicos que analisam o crescimento inicial de mudas de E. grandis (FERREIRA, 1994; GOMES et al., 1982), é importante considerar fatores que se distinguem entre os experimentos, tais como o ambiente, o clima, o substrato, a frequência de irrigação e a tecnologia de produção, entre outros.

A variável diâmetro do colo apresentou grande incremento (duas vezes) e resposta quadrática ao uso de adubação. As mudas submetidas à adubação com FLL tiveram um crescimento máximo de 2,62 mm de diâmetro quando submetidas à dosagem de $8 \mathrm{~km} \cdot \mathrm{m}^{-3}$. As doses de 6 e $10 \mathrm{~kg} \cdot \mathrm{m}^{-3}$ apresentaram, respectivamente, um DC de 2,34 e 2,54 mm, sendo o menor valor 1,06 mm, verificado no tratamento sem adição de FLL (testemunha). Quando calculada a dose de máxima eficiência técnica, a dosagem máxima obtida foi de $10,6 \mathrm{~kg} \cdot \mathrm{m}^{-3}$.

Binotto (2007) aponta que, entre as variáveis avaliadas, o DC é o parâmetro mais propício para indicar qualidade de muda, devido ao seu maior grau de relação com o IQD. Segundo Fonseca et al. (2002), a relação altura da parte aérea/diâmetro do coleto deve ser utilizada em conjunto com outros parâmetros na determinação do melhor padrão de qualidade das mudas.

Comparando entre si os tratamentos com adição de FC, podemos perceber que o maior DC apresentou-se na dose de $8 \mathrm{~kg} . \mathrm{m}^{-3}$ de FC. Esse resultado é semelhante ao tratamento que recebeu $4 \mathrm{~kg} . \mathrm{m}^{-3}$ de FLL. 
Aumento na biomassa fresca da parte aérea (BFPA) em mais de 8 vezes com uso de FLL confirma a necessidade de adubação. Os acréscimos ocorreram até uso de $6 \mathrm{~kg} \cdot \mathrm{m}^{-3}$ de FLL, quando houve estabilização. O acréscimo observado é bastante significativo se comparado ao tratamento sem adição de FLL, sendo que não há diferenciação entre os três tratamentos com maior incremento de BFPA. Por outro lado, quando as mudas são fertilizadas com FC, a dose que melhor resultou em produção de BFPA foi $10 \mathrm{~kg} . \mathrm{m}^{-3}$ de FC (Figura 2).

Nas variáveis biomassa seca da parte aérea (BSPA), biomassa seca da raiz (BSR) e biomassa seca total (BST), verificou-se que as doses de FLL influenciaram a espécie em questão, sendo que as máximas médias em valor absoluto, para as três variáveis, corresponderam ao tratamento com $10 \mathrm{~kg} . \mathrm{m}^{-3}$ de FLL, alcançando 1,392 g/planta para BSPA, 0,593 g/planta para BSR e 1,985 g/planta para BST.

Esses resultados se opõem aos levantados por Lang (2008) na avaliação de desenvolvimento de mudas de ipê-roxo (Tabebuia avellanedae) e angico-branco (Anadenanthera colubrina) submetidos a diferentes dosagens de FLL, o que leva a concluir que as diferentes dosagens não influenciaram de maneira significativa as variáveis BSPA, BSR e BST.
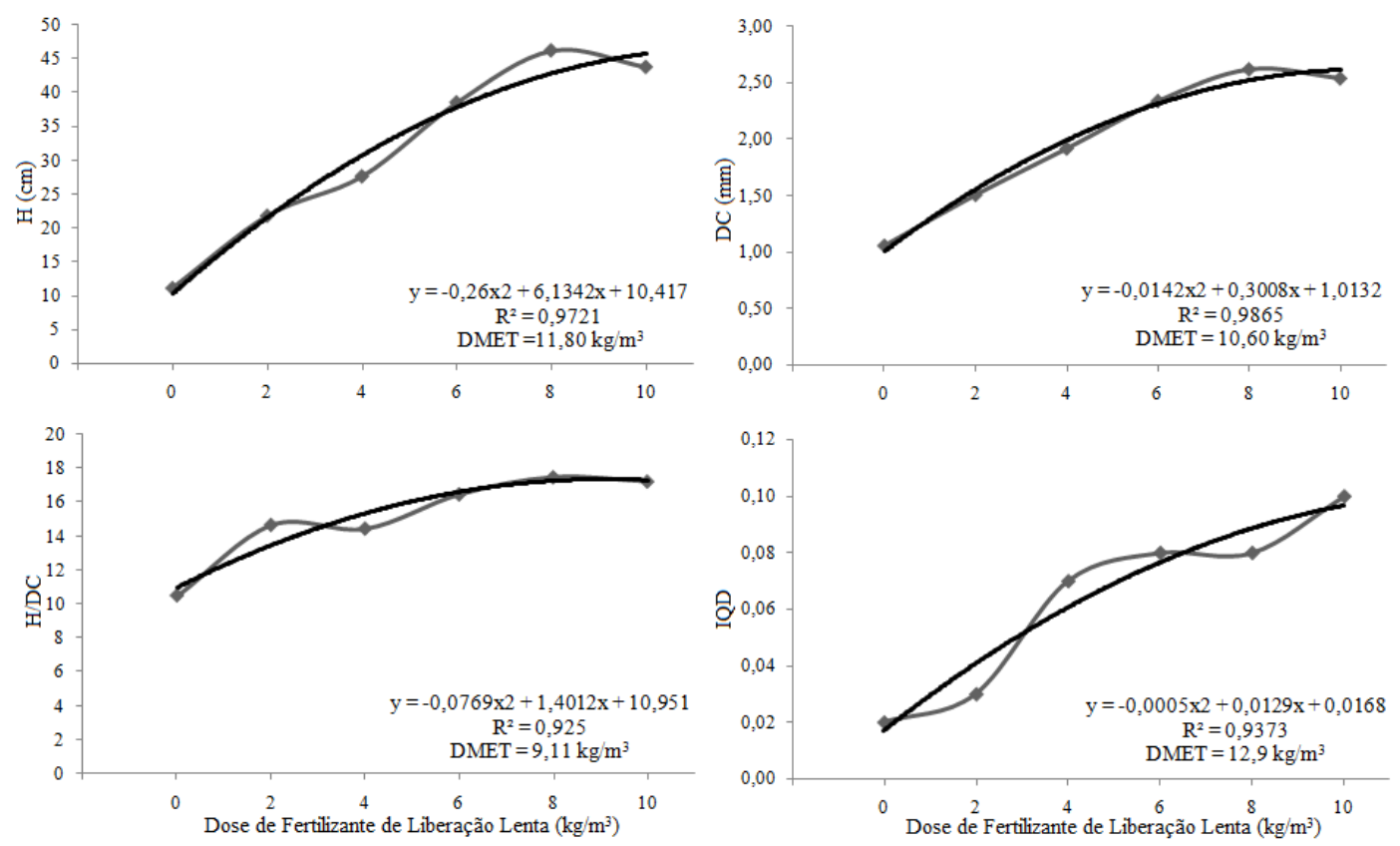

Figura 1. Dose de máxima eficiência técnica para as variáveis altura da parte aérea (H), diâmetro do colo (DC), relação (H/DC) e índice de qualidade de Dickson (IQD), em função de dose de fertilizante de liberação lenta das mudas de Eucalyptus grandis.

Figure 1. Maximum level of technical efficiency for the variables ground part height, collar diameter, height and collar diameter ratio and Dickson quality index according to controlled-release fertilizer doses of Eucalyptus grandis seedling.

Em relação ao BSPA, resultados semelhantes foram levantados por Silveira et al. (1995), ao analisar mudas de sementes de Eucalyptus grandis na fase de expedição aos 97 dias de idade, produzidas com substrato à base de casca de pínus, apontando que o peso da BSPA varia entre 1,2 e 1,6 g por planta.

Gomes e Paiva (2004) ratificam que o peso de BSPA indica a rusticidade e correlaciona-se diretamente com a sobrevivência e desempenho inicial das mudas após o plantio em campo.

Mendonça et al. (2008), trabalhando com Tamarindus indica L., observaram resultados semelhantes na utilização de FLL em diferentes doses e ambientes para a produção de mudas de tamarindeiro, encontrando a DMET próxima a $9 \mathrm{~kg} . \mathrm{m}^{-3}$ de substrato para esse parâmetro (BSPA).

Sgarbi et al. (1999) avaliaram a produção de mudas de eucalipto (Eucalyptus urophylla) sob a influência da aplicação de FLL e concluíram que a maior produção de biomassa seca da parte aérea foi

FLORESTA, Curitiba, PR, v. 45, n. 1, p. 85 - 96, jan. / mar. 2015.

Rossa, U. B. et al.

ISSN eletrônico 1982-4688 / ISSN impresso 0015-3826

DOI: $10.5380 /$ rf.v45i1.31224 
obtida nos tratamentos 2 e $4 \mathrm{~kg} . \mathrm{m}^{-3}$, resultados esses diferentes dos apurados neste trabalho, em que as doses com 6,8 e $10 \mathrm{~kg} \cdot \mathrm{m}^{-3}$ de FLL foram as que produziram maior peso de BSPA.

Avaliando outra espécie de eucalipto (Eucalyptus globulus subsp. Maidenii) sob fertilização NPK, Pezzutti et al.(1999) concluíram que os máximos crescimentos em altura, diâmetro do colo, massa seca da parte aérea e massa seca do sistema radicular são obtidos no intervalo de doses de 6,7 a $7,5 \mathrm{~kg} \cdot \mathrm{m}^{-3}$ de substrato. Esses resultados corroboram os levantados no presente estudo, no qual a melhor produção desses mesmos parâmetros ficou estabelecida quando houve fertilização com dose de $8 \mathrm{~kg} \cdot \mathrm{m}^{-3}$.

Para esses três parâmetros, verificam-se resultados semelhantes para as mudas fertilizadas com FC, com as melhores produções de BSPA, BSR e BST ocorreram com as maiores doses de FC, correspondentes a 6,8 e $10 \mathrm{~kg} . \mathrm{m}^{-3}$, exceto BST e BSR das mudas de T10, que apresentaram menor peso (Figura 2).

Para a variável biomassa seca da raiz, Brondani et al. (2008) observaram comportamento linear decrescente, com uma redução de aproximadamente $30 \%$ em relação à testemunha na dose de $5 \mathrm{~kg} \cdot \mathrm{m}^{-3} \mathrm{de}$ fertilizante.
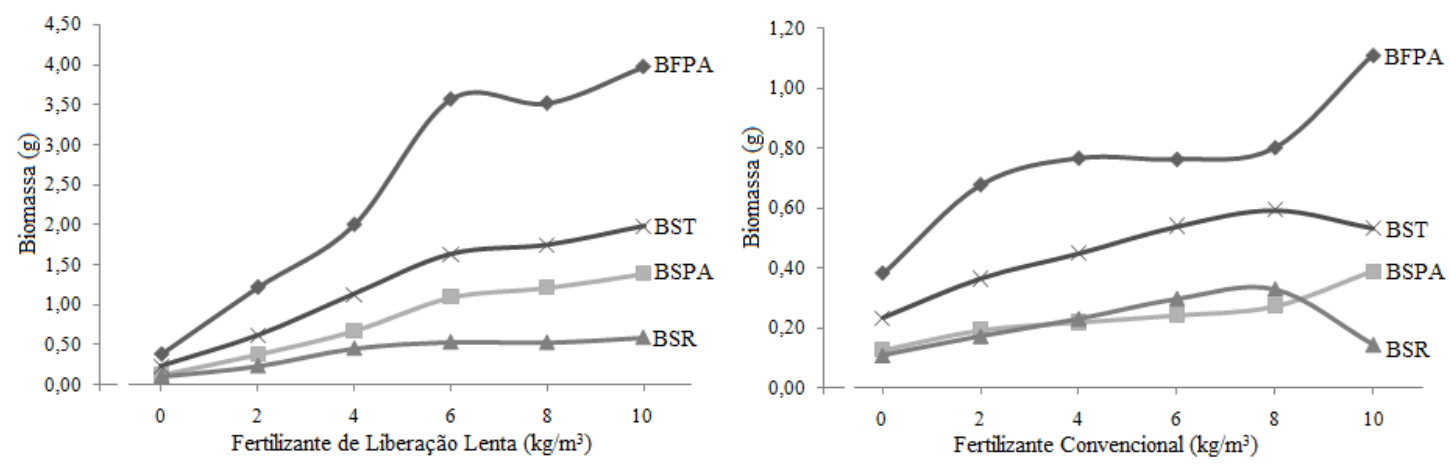

Figura 2. Curvas de tendência para as variáveis biomassa fresca da parte aérea (BFPA), biomassa seca total (BST), biomassa seca da parte aérea (BSPA) e biomassa seca da raiz (BSR) em função da dose de fertilizante de liberação lenta e fertilizante convencional em mudas de Eucalyptus grandis.

Figure 2. Curves of trend for the variable fresh biomass of the aerial part (BFPA), total dry biomass (BST), dry biomass of the aerial part (BSPA), dry biomass of the root (BSR) dry biomass of the root (BSR) in function of doses of slow release and conventional fertilizer in Eucalyptus grandis seedlings.

Segundo Almeida et al. (2005), a biomassa radicial proporciona melhor desempenho das plantas quando transferidas para o campo, por apresentarem maior capacidade de sustentação e absorção de água e nutrientes.

Binotto (2007) estudou a relação entre as variáveis de crescimento e o índice de qualidade de Dickson em eucalipto (Eucalyptus grandis) e pínus (Pinus elliottii) e concluiu que, independentemente da espécie avaliada, a massa seca de raiz é a variável que apresentou maior efeito direto e indireto nas demais variáveis, afetando o IQD. Assim, constitui-se na principal variável a ser estudada e a que indica realmente a qualidade da muda. Portanto, analisando essa variável no presente estudo, pode-se inferir que a dose de $12,9 \mathrm{~kg} . \mathrm{m}^{-3}$ é a mais indicada, quando se busca a robustez do coleto nas mudas (Figura 1).

Doses crescentes do FLL na formulação 15-09-12 resultaram em melhor qualidade das mudas de cafeeiro, até as doses que maximizaram os resultados para parte aérea $\left(7,7\right.$ a $\left.9,1 \mathrm{~kg} \cdot \mathrm{m}^{-3}\right)$ e sistema radicular $\left(6,8 \mathrm{~kg} . \mathrm{m}^{-3}\right)$, apontam Barbizan et al. (2002).

Ao analisar os resultados de índices de qualidade, notam-se que os tratamentos que apresentaram maior influência foram os três que tiveram as maiores doses, exceto para a relação H/DC, em que T2 e T3 foram iguais estatisticamente aos outros com maiores doses de FLL.

Para José et al. (2009), as medidas de diâmetro e altura são as principais características para seleção de mudas para o plantio. Segundo preconiza Carneiro (1995), a relação H/DC exprime o equilíbrio de desenvolvimento das mudas, pois conjuga duas importantes características em apenas um só 
índice, e quanto menor for seu valor, melhor a qualidade da muda, consequentemente, maior a capacidade de sobrevivência e estabelecimento no local de plantio definitivo. Entretanto há a necessidade de calibração desses índices para cada espécie, através de experimentos que avaliem a qualidade da muda e a relacione com seu desenvolvimento em campo.

Os índices H/D e IQD para espécies florestais devem ser menor que 10 e maior que 0,2, respectivamente, para que a muda apresente alta qualidade e, consequentemente, alta sobrevivência após o plantio (HUNT, 1990).

Estabelecendo-se como padrão para o índice de Dickson o valor mínimo de 0,20, conforme recomendação de Hunt (1990), observa-se que em nenhuma das doses de FLL e FC as mudas atingiram esse valor. Entretanto, na avaliação da qualidade das mudas, não se devem utilizar, isoladamente, parâmetros morfológicos e suas relações, para não se correr o risco de selecionar mudas mais altas, porém fracas, descartando as menores, mas com maior vigor (FONSECA et al., 2002).

Conforme Fonseca (2000), o manejo das mudas no viveiro pode permitir que se atinja o valor mínimo desejado. Avaliando a relação entre as variáveis de crescimento e o índice de qualidade de Dickson em mudas de eucalipto (Eucalyptus grandis) e pínus (Pinus elliottii), Binotto (2007) concluiu que o IQD foi eficiente para indicar qualidade de mudas, pois se apresentou bastante relacionado com as variáveis estudadas para as duas espécies. Vale ressaltar que ensaio de calibração do IQD para as espécies de interesse configura uma importante necessidade para as investigações de qualidade de muda.

Binotto (2007), em estudo com Eucaliptus grandis para verificar a relação e a eficiência entre variáveis biométricas de crescimento e a interferência que elas exercem sobre o índice de qualidade de Dickson, obteve IQD de 0,05 aos 120 dias de emergência, resultados semelhantes aos encontrados no presente trabalho.

A dose máxima eficiência técnica (DMET) é distinta dentro dos diferentes parâmetros avaliados, nos tratamentos com fertilização com FLL. A DMET para altura $(\mathrm{H})$ foi de $11,8 \mathrm{~kg} . \mathrm{m}^{-3}$ de FLL. Para a variável diâmetro de coleto (DC), estimou-se uma DMET de $10,6 \mathrm{~kg} \cdot \mathrm{m}^{-3}$ de FLL. Para a obtenção da melhor relação altura e diâmetro de coleto da muda (H/DC), estimou-se uma DMET de $9,1 \mathrm{~kg} . \mathrm{m}^{-3} \mathrm{de}$ FLL. Avaliando o índice de qualidade de Dickson (IQD), estimou-se uma DMET de $12,9 \mathrm{~kg} . \mathrm{m}^{-3}$ de FLL.

Moraes Neto et al. (2003a), também em trabalho de fertilização com FLL em mudas de mutambo (Guazuma ulmifolia), canafístula (Peltophorum dubium) e eucalipto (Eucalyptus grandis), recomendam a utilização de $6,4 \mathrm{~kg} \cdot \mathrm{m}^{-3}$ de Basacote no substrato, resultado com recomendações de doses abaixo do presente estudo. Ainda Moraes Neto et al. (2003b), em trabalho com mutambo (Guazuma ulmifolia), capixingui (Croton floribundus), canafístula (Peltophorum dubium), pau-d'alho (Gallesia integrifolia) e cabreúva (Myroxylon peruiferum), concluíram que doses de FLL entre 3,2 e 4,8 kg.m foram as que resultaram em mudas de melhor qualidade para todas as espécies estudadas.

\section{Avaliação nutricional}

A concentração dos nutrientes obedeceu à ordem $\mathrm{N}>\mathrm{K}>\mathrm{Ca}>\mathrm{Mg}>\mathrm{P}$, para as mudas dos tratamentos com FLL (T5 e T6) que melhor representaram a qualidade das mudas (entre 9,1 e 12,9 kg.m ${ }^{-3}$ ) (Tabela 2).

$\mathrm{O} \mathrm{Mn}$ foi o micronutriente com maior concentração, seguido do $\mathrm{Zn}, \mathrm{Fe}$ e $\mathrm{Cu}$, nessa mesma faixa de dosagem de FLL.

Quando do uso de FC na maior dose, permitindo máximo crescimento, houve mudança na ordem dos nutrientes em relação ao FLL, a saber, $\mathrm{K}>\mathrm{P}>\mathrm{Ca}>\mathrm{N}>\mathrm{Mg}$, mas a sequência para micronutriente foi a mesma. Tal diferença pode explicar o menor índice de crescimento observado com FC, especialmente considerando valores muito mais elevados de $\mathrm{P}$ em relação a $\mathrm{N}$.

Resultados semelhantes a esses foram apontados por Silveira (2000), ao verificarem as quantidades extraídas dos nutrientes em brotação de dois clones de Eucalyptus, no período entre 7 e 16 dias de idade, na condição de minijardim clonal hidropônico aberto. A concentração dos nutrientes nas brotações obedeceu à seguinte ordem decrescente para ambos os clones estudados: $\mathrm{N}>\mathrm{K}>\mathrm{P}>\mathrm{Ca}>\mathrm{Mg}>\mathrm{S}$, sendo que para os micronutrientes a ordem de concentração dos elementos foi $\mathrm{Mn}>\mathrm{Fe}>\mathrm{Zn}>\mathrm{B}>\mathrm{Cu}$. Essa mesma sequência dos micronutrientes corroboram os resultados encontrados por Silveira et al. (1995), ao avaliar a absorção de micronutrientes por mudas de Eucalyptus grandis em condição de viveiro, aos 97 dias de idade.

As concentrações de C, N, K, Mg e Zn na planta, independentemente do tipo de adubo aplicado, não sofreram alterações, contrastando com o aumento no crescimento. Ainda, a concentrações observadas

FLORESTA, Curitiba, PR, v. 45, n. 1, p. 85 - 96, jan. / mar. 2015.

Rossa, U. B. et al.

ISSN eletrônico 1982-4688 / ISSN impresso 0015-3826

91

DOI: $10.5380 /$ rf.v45i1.31224 
quando do uso de FC e FLL para os mesmos elementos são próximas. Logo, tais nutrientes provavelmente não estão associados a variação na produtividade em função das doses e entre as diferentes fontes de fertilizante.

Em estudo de monitoramento nutricional e fertilização em macro, mini e microjardim clonal de Eucalyptus, Higashi et al. (2000) indicam teores de macro e micronutrientes considerados adequados para altas taxas de enraizamento das miniestacas, destacando-se que doses menores que $22 \mathrm{~g} \cdot \mathrm{kg}^{-1} \mathrm{de} \mathrm{N}$, 1,5 g.kg ${ }^{-1}$ de P, 15 g. $\mathrm{kg}^{-1}$ de K, 4 g.. $\mathrm{kg}^{-1}$ de Ca e $1,5 \mathrm{~g} \cdot \mathrm{kg}^{-1}$ de $\mathrm{Mg}$ podem ser consideradas deficientes. Comparativamente ao presente trabalho, apenas o $\mathrm{N}$ e o $\mathrm{K}$ encontram-se abaixo desses níveis, em mudas fertilizadas com FLL e FC, sendo que a concentração de P em mudas fertilizadas com doses de FC apresentou níveis considerados altos pelo supracitado autor. Em outro trabalho, Higashi et al. (1999) avaliaram os teores de macro e micronutrientes na parte aérea de Eucalyptus grandis, encontrando teores maiores que $17 \mathrm{~g} . \mathrm{kg}^{-1}$ para N, 2,1 g. $\mathrm{kg}^{-1}$ para $\mathrm{P}$ e acima de $7,2 \mathrm{~g} \cdot \mathrm{kg}^{-1}$ para K. Para o $\mathrm{P}$, apenas para as mudas dos tratamentos com FC foram verificados teores acima desses valores, entretanto, para o $\mathrm{K}$, os valores dos teores dos tratamentos com FLL e FC podem ser considerados parecidos, acima da faixa de $7,2 \mathrm{~g} \cdot \mathrm{kg}^{-1}$.

A concentração de $\mathrm{P}$, partindo de valor elevado na testemunha, apresentou decréscimo seguido de aumento nas últimas doses, quando do uso de FLL, mas se manteve constante quando do uso de FC. Logo, as concentrações do mesmo também não podem explicar o grande aumento na produtividade observada no FLL. Contudo, tal fato pode estar relacionado ao efeito diluição, pelo qual grande aumento na produtividade faz com que haja diminuição da concentração de alguns elementos. A resposta quadrática às doses de fertilizantes combina com diminuição e retorno aos níveis normais observados para o P quando do uso de FLL.

A concentração de Pé a única que combina condições que possam explicar a grande diferença de produtividade entre as diferentes fontes. Primeiro, porque os valores de $\mathrm{P}$ são muito mais elevados no FC do que FLL, sugerindo elevada disponibilização já nas primeiras doses do FC. Segundo, porque os valores observados no FC estão, em geral, 5 vezes mais elevados do que possa ser considerado normal. Terceiro, porque o valor elevado de $\mathrm{P}$ obtido na testemunha sugere que em algumas condições pode haver concentração de $\mathrm{P}$, dada a carência e o pequeno crescimento. $\mathrm{O}$ excesso de $\mathrm{P}$ pode comprometer a nutrição da planta ao interagir de maneira antagônica com $\mathrm{Zn}, \mathrm{Fe}, \mathrm{Mn}$ e $\mathrm{Cu}$, sem necessariamente decréscimo na concentração de nutrientes dos mesmos na planta.

A concentração de $\mathrm{Mn}$ apresentou relação inversa quanto às doses aplicadas e, embora em ambos os adubos utilizados as concentrações de Mn observadas nas doses mais altas tenham sido elevadas, elas não comprometeram a produtividade. Contudo, as concentrações de Mn obtidas entre as diferentes fontes são próximas, não podendo ser utilizadas para explicar a grande diferença de crescimento proporcionada pelas mesmas.

O comportamento da concentração de Fe na planta partiu de valores normais, decresceu para valores que podem ser considerados muito baixos e elevou-se novamente, para ambas as fontes de fertilizantes. Tal comportamento não era esperado e contrasta com aumento de crescimento, o que leva a concluir que possivelmente não esteja relacionado ao crescimento. Os valores observados para $\mathrm{Fe}$ na planta quando do uso de FC e FLL são próximos, não podendo ser utilizados para justificar a grande diferença de produtividade obtida entre as diferentes fontes.

As maiores quantidades de carbono (C) foram superiores nas mudas dos tratamentos que receberam FLL, sendo que a dose de $8 \mathrm{~kg} \cdot \mathrm{m}^{-3}$ de FLL foi a que mais proporcionou produção desse elemento, com 435,9 g. $\mathrm{kg}^{-1}$. As mudas produzidas com $6 \mathrm{~kg} \cdot \mathrm{m}^{-3}$ de $\mathrm{FC}$ foi a que proporcionou maior produção de $\mathrm{C}$, com $420,5 \mathrm{~g} \cdot \mathrm{kg}^{-1}$.

Os tratamentos com maior concentração de nitrogênio $(\mathrm{N})$ foram os que receberam doses de 2 kg.m ${ }^{-3}$ de FC e 8 kg.m ${ }^{-3}$ de FLL, com quantitativos de $9,22 \mathrm{~g} \cdot \mathrm{kg}^{-1}$ e $8,42 \mathrm{~g}^{-\mathrm{kg}^{-1}}$, respectivamente.

As concentrações de $\mathrm{P}, \mathrm{K}, \mathrm{Ca}, \mathrm{Mg}$ e $\mathrm{Zn}$ na parte aérea das mudas produzidas com FC apresentam teores maiores quando comparados às produzidas com FLL, contrastando com produtividade.

Em experimento de campo, objetivando avaliar o melhor fertilizante para produção de Eucalyptus grandis, Poggiani et al. (2000) encontraram concentrações significativamente superiores de $\mathrm{P}$ nas folhas dos tratamentos com FC em comparação com os adubados com biossólido, sugerindo a alta disponibilidade de $\mathrm{P}$ nos fertilizantes minerais de pronta liberação. Há uma similaridade entre o comportamento dessa espécie e o tipo de fertilizante utilizado: de um lado a sugestão da alta capacidade de extração de $\mathrm{P}$ e de outro a característica de pronta disponibilidade desse macronutriente, encontradas nos FC, traduzidas pelos teores de $\mathrm{P}$ na parte aérea de mudas produzidas com esse tipo de fertilizante. 
Tabela 2. Composição química da parte aérea de mudas de Eucalyptus grandis produzido sob doses de fertilizante de liberação lenta e de fertilizante convencional.

Table 2. Chemical composition of the foliage of Eucalyptus grandis seedlings produced under doses of slow release fertilizer and conventional fertilizer.

\begin{tabular}{|c|c|c|c|c|c|c|c|c|c|c|}
\hline \multirow{2}{*}{ Dose/Tratamento } & $\mathbf{C}$ & $\mathbf{N}$ & $\mathbf{P}$ & $\mathbf{K}$ & $\mathbf{C a}$ & Mg & $\mathbf{F e}$ & Mn & $\mathbf{C u}$ & Zn \\
\hline & \multicolumn{6}{|c|}{ 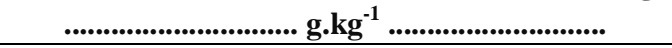 } & \multicolumn{4}{|c|}{................. mg.kg mg $^{-1} \ldots \ldots . . . . . . . . .}$. \\
\hline \multicolumn{11}{|c|}{ Composição química com fertilizante de liberação lenta - FLL } \\
\hline 0 kg.m ${ }^{-3}$ FLL $(\mathrm{T} 1)$ & 409 & 7,82 & 5,40 & 8,50 & 10,53 & 4,15 & 77,0 & 122,0 & 17,0 & 69,0 \\
\hline 2 kg.m ${ }^{-3}$ FLL (T2) & 433 & 6,41 & 0,80 & 13,20 & 7,58 & 2,90 & 81,0 & 149,0 & 9,0 & 54,0 \\
\hline 4 kg.m ${ }^{-3}$ FLL (T3) & 431 & 5,55 & 0,57 & 8,50 & 6,25 & 2,13 & 5,0 & 173,0 & 2,0 & 36,0 \\
\hline 6 kg.m ${ }^{-3}$ FLL (T4) & 425 & 6,96 & 0,74 & 5,80 & 4,88 & 2,25 & 5,0 & 239,0 & 1,0 & 36,0 \\
\hline 8 kg.m ${ }^{-3}$ FLL (T5) & 436 & 8,42 & 1,56 & 7,70 & 5,68 & 2,50 & 37,0 & 254,0 & 5,0 & 39,0 \\
\hline $10 \mathrm{~kg} \cdot \mathrm{m}^{-3} \mathrm{FLL}(\mathrm{T} 6)$ & 425 & 7,79 & 1,73 & 6,70 & 5,28 & 2,58 & 45,0 & 308,0 & 1,0 & 51,0 \\
\hline \multicolumn{11}{|c|}{ Composição química com fertilizante convencional - FC } \\
\hline $0 \mathrm{~kg} \cdot \mathrm{m}^{-3} \mathrm{FC}(\mathrm{T} 1)$ & 409 & 7,82 & 5,40 & 8,50 & 10,53 & 4,15 & 77,0 & 122,0 & 17,0 & 69,0 \\
\hline $2 \mathrm{~kg} \cdot \mathrm{m}^{-3} \mathrm{FC}(\mathrm{T} 2)$ & 415 & 9,22 & 12,72 & 15,00 & 8,85 & 3,60 & 71,0 & 123,0 & 11,0 & 57,0 \\
\hline $4 \mathrm{~kg} \cdot \mathrm{m}^{-3} \mathrm{FC}(\mathrm{T} 3)$ & 416 & 8,35 & 12,28 & 8,80 & 10,20 & 3,50 & 48,0 & 132,0 & 4,0 & 60,0 \\
\hline $6 \mathrm{~kg} \cdot \mathrm{m}^{-3} \mathrm{FC}(\mathrm{T} 4)$ & 420 & 6,42 & 11,96 & 14,40 & 9,18 & 3,18 & 1,0 & 236,0 & 2,0 & 49,0 \\
\hline $8 \mathrm{~kg} \cdot \mathrm{m}^{-3} \mathrm{FC}(\mathrm{T} 5)$ & 416 & 6,68 & 11,88 & 12,90 & 9,48 & 3,58 & 13,0 & 361,0 & 4,0 & 57,0 \\
\hline $10 \mathrm{~kg} \cdot \mathrm{m}^{-3} \mathrm{FC}(\mathrm{T} 6)$ & 417 & 6,50 & 11,20 & 13,50 & 9,73 & 2,90 & 33,0 & 561,0 & 8,0 & 52,0 \\
\hline
\end{tabular}

Wadt et al. (1998), ao analisarem tecido vegetal de um híbrido de E. grandis x E. urophylla, estabeleceram uma faixa ótima de concentração dos macronutrientes nas diferentes partes da planta adulta. Ao comparar os resultados alcançados por esses autores com os apurados no presente trabalho, pode-se inferir que os níveis de macronutrientes $\mathrm{Ca}, \mathrm{Mg}$ e $\mathrm{P}$ considerados ótimos encontram-se abaixo dos teores dos tecidos da parte aérea das mudas produzidas com FLL e FC. Entretanto, são inferiores aos teores de $\mathrm{N}$ das mudas adubadas com FC e FLL, sendo que os teores de K encontram-se dentro da faixa considerada ótima, mencionada por aqueles autores.

\section{CONCLUSÕES}

- Mudas de Eucalyptus grandis apresentaram melhores padrões de qualidade quando da utilização do fertilizante de liberação lenta em comparação com o fertilizante convencional.

- Melhores padrões de mudas foram verificados sob doses entre 9,1 e $12,9 \mathrm{~kg} \cdot \mathrm{m}^{-3}$ de fertilizante de liberação lenta, tendo a concentração de nutrientes a ordem $\mathrm{N}>\mathrm{K}>\mathrm{Ca}>\mathrm{Mg}>\mathrm{P}>\mathrm{Mn}>\mathrm{Zn}>\mathrm{Fe}>\mathrm{Cu}$.

\section{REFERÊNCIAS}

ALMEIDA, L. S. de; MAIA, N.; ORTEGA, A. R.; ÂNGELO, A. C. Crescimento de mudas de Jacarandá puberula Cham. em viveiro, submetidas a diferentes níveis de luminosidade. Ciência Florestal, v. 15, n. 3, p. $323-329,2005$.

BARBIZAN, E. L.; LANA, R. M. Q.; MENDONÇA, F. C.; MELO, B.; SANTOS, C. M.; MENDES, A. F. Produção de mudas de cafeeiro em tubetes associada a diferentes formas de aplicação de fertilizantes. Ciência Agrotécnica, Lavras edição Especial, p. 1471 - 1480, dezembro, 2002.

BENNETT, E. Slow-release fertilizers. Virginia Gardener Newsletter, Blacksburg, v. 11, n. 4., 1996. Disponível em: <www.ext.vt.edu/departments/envirohort/articles/misc/slowrels.html>. Acesso em: 27/04/2009.

BINOTTO, A. F. Relação entre variáveis de crescimento e o índice de qualidade de Dickson em mudas de Eucalyptus grandis W. Hill ex Maid e Pinus elliottii var. elliottii - Engelm. 2007. $53 \mathrm{f}$. Dissertação (Mestrado em Engenharia Florestal) - Universidade Federal de Santa Maria, Santa Maria, RS, 2007.

BOCKMAN, O. C.; OLFS, H. W. Fertilizers, agronomy and $\mathrm{N}_{2} \mathrm{O}$. Nutrient Cycling in Agroecosystems, Dordrecht, v. 52, n. 2/3, p. 165 - 170, 1998. 
BRONDANI, G. E.; SILVA, A. J. C.; REGO, S. S.; GRISI, F. A.; NOGUEIRA, A. C.; WENDLING, I.; ARAÚJO, M. A. Fertilização de liberação controlada no crescimento inicial de angico-branco. Scientia Agraria, Curitiba, v. 9, n. 2, p. 167 - 176, 2008.

CARNEIRO, J. G. de A. Produção e qualidade de mudas florestais. Curitiba: UFPR/FUPEF; Campos: UENF, 451 p, p. 57 - 92. 1995.

CECONI, D. E.; POLETTO, I.; LOVATO, T.; MUNIZ, M. F. B. Exigência nutricional de mudas de ervamate (Ilex paraguariensis A. St.-Hil.) à adubação fosfatada. Revista Ciência Florestal, Santa Maria, v. 17, n. 1, p. 25 - 32, 2007.

CSIZINSZKY, A. A. Yield response of ell epper and tomato to controlled-release fertilizers on sand. Journal of Plant Nutrition, v. 17, n. 9, p. 1535 - 1549, 1994.

DICKSON, A.; LEAF, A. L.; HOSNER, J. F. Quality appraisal of white spruce and white pine seedling stock in nurseries. Forest Chronicle, v. 36, p. 10 - 13, 1960.

FERREIRA, M. G. R. Crescimento de mudas de Eucalyptus grandis Hill ex Maiden, em resposta a tamanhos de embalagem, substratos e fertilização NPK. Viçosa: UFV, 1994. 44 p. Dissertação (Mestrado Ciência Florestal) - Universidade Federal de Viçosa, 1994.

FONSECA, E. P. Padrão de qualidade de mudas de Trema micrantha (L.) Blume., Cedrela fissilis Vell. e Aspidosperma polyneuron Müll. Arg. produzidas sob diferentes períodos de sombreamento. 2000. 113 f. Tese (Doutorado em Ciências Agrárias) - Universidade Estadual Paulista, Faculdade de Ciências Agrárias e Veterinárias, Jaboticabal, SP, 2000.

FONSECA, E. P.; VALERI, S. V.; MIGLIORANZA, E.; FONSECA, N. A. N.; COUTO, L. Padrão de qualidade de mudas de Trema micrantha (L.) Blume. produzidas sob diferentes períodos de sombreamento. Revista Árvore. Viçosa, MG, v. 26, n. 4, p. 515 - 523, 2002.

FREITAS, T. A. S. de; BARROSO, D. G.; CARNEIRO, J. G. de A.; PENCHEL, R. M.; LAMÔNICA, K. R.; FERREIRA, D. A. Desempenho radicular de mudas de eucalipto produzidas em diferentes recipientes e substratos. Revista Árvore, Viçosa, v. 29, n. 6, p. 853 - 861, 2005.

GOMES, J. M.; COUTO, L.; LEITE, H. G.; XAVIER, A.; GARCIA, S. L. R. Parâmetros morfológicos na avaliação da qualidade de mudas de Eucalyptus grandis. Revista Árvore, Viçosa, v. 26, n. 6, p. 655 664, 2002.

GOMES, J. M.; BARROS, N. F. de; NEVES, J. C. L. Métodos de aplicação de adubo em diferentes solos para produção de mudas de Eucalyptus grandis W. Hill ex Maiden. Revista Árvore, Viçosa, v. 6, n. 1, p. $52-63,1982$.

GOMES, J. M.; PAIVA, H. N. Viveiros florestais - propagação sexuada. 3. ed. 116 p. Viçosa: UFV, 2004.

GONÇALVES, J. L.; POGGIANI, F. Substratos para produção de mudas florestais. In: SUELO CONGRESSO LATINO-AMERICANO DE CIÊNCIA DO SOLO. 1996, Águas de Lindoia, SP. Anais CD-ROM. Águas de Lindoia: SLCS:SBCS:ESALQ/USP:CEA - ESALQ/USP, 1996.

HEFNER S. G.; TRACY P. W.; The effect of nitrogen quantity and application timing on furrowirrigated rice. Journal of Production Agriculture, v. 4, n. 4, p. 541 - 546, 1991.

HIGASHI, E. N.; SILVEIRA, R. L. V. A.; MOREIRA, A. Relatório de assessoria e pesquisa na siderúrgica Barra Mansa (1997-1998). Piracicaba: IPEF, 1999. 77 p. (Relatório interno do IPEF)

HIGASHI, E. N.; SILVEIRA, R. L. V. A.; GONÇALVES, A. N. Monitoramento nutricional e fertilização em macro, mini e microjardim clonal de eucaliptos. In: GONÇALVES, J. L. M. e BENEDETTI, V. (eds.) Nutrição e fertilização florestal. Cap. 6, p. 191 - 218. Piracicaba: IPEF, 2000.

HUNT, G. A. Effect of styroblock design and cooper treatment on morphology of conifer seedlings. IN: TARGET SEEDLIN ASSOCIATINS, GENERAL TECHNICAL REPORT RM-200. 1990. Roseburg: Proceedings... Fort Collins: United States Department of Agriculture, Forest Service, 1990. p. 218 - 222. 
JOSÉ, A. C.; DAVIDE, A. C.; OLIVEIRA, S. L. Efeito do volume do tubete, tipo e dosagem de adubo na produção de mudas de aroeira (Schinus terebinthifolia Raddi). Agrarian, v. 2, n. 3, p.73 - 86, 2009.

JOSÉ, A. C.; DAVIDE, A. C.; OLIVEIRA, S. L. Produção de mudas de aroeira Schinus terebinthifolia Raddi para recuperação de áreas degradadas pela mineração de bauxita. Cerne, v. 11, n. 2, p. 187 - 203, 2005.

LANG, A. Efeitos da aplicação de fertilizante de liberação lenta no estabelecimento de mudas de ipê-roxo e angico-branco em área de domínio ciliar. 1998. 70 f. Dissertação (Mestrado em Agronomia) - Universidade Estadual do Oeste do Paraná - UNIOESTE, Marechal Cândido Rondon, PR, 2008.

MARQUES, V. B. Efeito de fontes e doses de nitrogênio sobre o crescimento de angico-vermelho (Anadenanthera macrocarpa (Benth.) Brenan), Jacarandá-da-bahia (Dalbergia nigra (Vell.) Fr. All. ex Benth.) e sabiá (Mimosa caesalpiniaefolia Benth.). 2004. 97 p. Dissertação (Mestrado em Ciência Florestal) - Universidade Federal de Viçosa, Viçosa, 2004.

MARSCHNER, H. Mineral nutrition of higher plants. London: Academic, 1995. 889 p.

MARTINS, A. P. L.; REISSMANN, C. B. Material vegetal e as rotinas laboratoriais nos procedimentos químico-analíticos. Scientia Agraria, Curitiba, v. 8, n. 1, p. 1 - 17, 2007.

MENDONÇA, D.; ABREU, N. A. A.; SOUZA, H. A.; TEIXEIRA, G. A.; HAFLE, O. M.; RAMOS, J. D. Diferentes ambientes e Osmocote ${ }^{\circledR}$ na produção de mudas de tamarindeiro (Tamarindus indica). Ciência e Agrotecnologia, Lavras, v. 32, n. 2, p. 391 - 397, 2008.

MEXAL, J. L.; LANDIS, T. D. Target seedling concepts: height and diameter. In: TARGET SEEDLING SYMPOSIUM, MEETING OF THE WESTERN FOREST NURSERY ASSOCIATIONS, GENERAL TECHNICAL REPORT RM-200, 1990, Roseburg. Proceedings... Fort. Collins: United States Department of Agriculture, Forest Service, 1990. p. 17 - 35.

MORAES NETO, S. P. GONÇALVES, J. L. M.; ARTHUR JR., J. C.; DUCATTI, F.; AGUIRRE JR., J. H. Fertilização de mudas de espécies arbóreas e nativas exóticas. Revista Árvore. v. 27, n. 2, p. 129 - 137. 2003a.

MORAES NETO, S. P.; GONÇALVES, J. L. M.; RODRIGUES, C. J.; GERES, W. L. A.; DUCATTI, F.; AGUIRRE JR., J. H. Produção de mudas de espécies arbóreas nativas com combinações de adubos de liberação controlada e prontamente solúveis. Revista Árvore, Viçosa, v. 27, n. 6, p. 779 - 789, 2003 b.

PARVIAINEN, J. V. Qualidade e avaliação de qualidade de mudas florestais. In: SEMINÁRIO DE SEMENTES E VIVEIROS FLORESTAIS, 1., 1981, Curitiba. Anais... Curitiba: FUPEF, 1981, p. 59 - 90.

PEZZUTTI, R. V.; SCHUMACHER, M. V.; HOPPE, J. M. Crescimento de mudas de Eucalyptus globulus em resposta à fertilização NPK. Ciência Florestal, v. 9, n. 2, p. 117 - 125, 1999.

POGGIANI, F.; GUEDES, M. C.; BENEDETTI, V. Aplicabilidade de biossólido em plantações florestais: I. reflexo no ciclo dos nutrientes. In: BETTIOL, W.; CAMARGO, O. A. (eds.). Impacto ambiental do uso agrícola do lodo de esgoto. Jaguariúna: Embrapa Meio Ambiente, 2000. p. 163 - 178.

REIS, M. G. F.; REIS, G. G. dos; REGAZZI, A. J.; LELES, P. S. S. Crescimento e forma de fuste de mudas de jacarandá-da-bahia (Dalbergia nigra Fr. Allem.) sob diferentes níveis de sombreamento e tempo de cobertura. Revista Árvore, v. 15, n. 1, p. 23 - 34, 1991.

SCIVITTARO, W. B.; OLIVEIRA, R. P.; RADMANN, E. B. Doses de fertilizante de liberação lenta na formação do porta-enxerto 'Trifoliata'. Revista Brasileira de Fruticultura, Jaboticabal, v. 26, n. 3, p. $520-523,2004$.

SGARBI, F.; SILVEIRA, R. L. V. A.; HIGASHI, E. N.; ANDRADE E PAULA, T.; MOREIRA, A.; RIBEIRO, F. A. Influência da aplicação de fertilizante de liberação controlada na produção de mudas de um clone de Eucalyptus urophylla. In: SIMPÓSIO SOBRE FERTILIZAÇÃO E NUTRIÇÃO FLORESTAL, 1., 1999, Piracicaba. Anais... Piracicaba, 1999. 4 p.

SHAVIV, A. Preparation methods and release mechanisms of controlled release fertilizers: agronomic efficiency and environmental significancy. Proceedings of the Fertiliser Society, York, UK, n. 41, p. 1 35, 1999.

FLORESTA, Curitiba, PR, v. 45, n. 1, p. 85 - 96, jan. / mar. 2015.

Rossa, U. B. et al.

ISSN eletrônico 1982-4688 / ISSN impresso 0015-3826

95

DOI: 10.5380/rf.v45i1.31224 
SILVEIRA, R. L. V. A. Efeito do potássio no crescimento, nas concentrações dos nutrientes e nas características da madeira juvenil de progênies de Eucalyptus grandis W. Hill ex Maiden cultivadas em solução nutritiva. Tese (Doutorado em Solos e Nutrição de Plantas) - ESALQ, Piracicaba, 2000.

SILVEIRA, R. L. V. A.; LUCA, E. F.; SHIBATA, F. Absorção de macronutrientes pelas mudas de Eucalyptus grandis em condição de viveiro. In: CONGRESSO BRASILEIRO DE CIÊNCIA DO SOLO, 25., Viçosa, 1995. Resumos expandidos. Viçosa: SBCS/UFV, p. 839 - 841, 1995.

TRUGILHO, P. F.; BIANCHI, M. L.; ROSADO, S. C. S.; LIMA, J. T. Qualidade da madeira de clones de espécies e híbridos naturais de Eucalyptus. Scientia Forestalis, Piracicaba, n. 73, p. 55 - 62, 2007.

WADT, P. G. S.; NOVAIS, R. F.; ALVAREZ, V. V. H.; FONSECA, S.; BARROS, N. F. Valores de referência para macronutrientes em eucalipto obtidos pelos métodos DRIS e CHANCE MATEMÁTICA. Revista Brasileira de Ciência do Solo, 22, p. 685 - 692, 1998. 